Acta vet. scand. $1974,15,274-282$.

From the Institute of Virology, Royal Veterinary College, and the National Veterinary Institute, Stockholm, Sweden.

\title{
EVALUATION OF SOME SEROLOGICAL TECHNIQUES FOR THE IDENTIFICATION OF MYCOPLASMA HYORHINIS AND MYCOPLASMA SUIPNEUMONIAE
}

\author{
By \\ Rolf Jansson
}

\begin{abstract}
JANSSON, ROLF: Evaluation of some serological techniques for the identification of Mycoplasma hyorhinis and Mycoplasma suipneumoniae. Acta vet. scand. 1974, 15, 274-282. - Eighteen strains of Mycoplasma hyorhinis and a strain of Mycoplasma suipneumoniae were tested in 4 serological tests, i. e., disc growth inhibition, metabolic inhibition, indirect haemagglutination and indirect epi-immunofluorescence. Only with immunofluorescence could all tested strains of M. hyorhinis be shown; no cross-reactions between M. hyorhinis and M. suipneumoniae could be detected. The other tests failed in many cases to identify strains of the same species, and they gave cross-reactions between M. hyorhinis and M. suipneumoniae.
\end{abstract}

porcine mycoplasmas; serological tests.

Several different serological techniques have been employed for the identification of M. hyorhinis and M. suipneumoniae, but they have not been evaluated in a comparative way, considering both these porcine species. In the present study the disc growth inhibition, metabolic inhibition, indirect haemagglutination, and epi-immunofluorescence have been compared using various strains of M.hyorhinis and a strain of M. suipneumoniae. Although these tests are all based on reactions between antibodies and mycoplasmal surface antigens, the results of the present study demonstrate the superiority of epi-immunofluorescence over the other techniques employed. 


\section{MATERIALS AND METHODS}

Mycoplasma strains. The strains of Mycoplasma hyorhinis and the M. suipneumoniae strain were kindly supplied by $Z$. Dinter of this Institute (F, FD, GDL, S, 200, K, S7, SK, G, W, and J), K. Bakos, National Veterinary Institute, Stockholm (KR and 378), N. F. Friis, State Veterinary Serum Laboratory, Copenhagen $(201,203,872$, and 859), T. Estola, State Veterinary Medical Institute, Helsinki (959), and J. G. Tully, National Institute of Allergy and Infectious Diseases, National Institutes of Health, Bethesda, Maryland (BTS-7). Dinter's strains were all "old" strains, passaged many times on culture media. The strains of Bakos, Friis, and Estola were all isolates with a low number of passages before testing. Stock cultures of all strains in liquid medium were stored at $-20^{\circ} \mathrm{C}$.

Mycoplasma media. The liquid medium consisted of beef-heart infusion, $40 \%(\mathrm{v} / \mathrm{v})$, Hanks' balanced salt solution with $0.2 \%(\mathrm{w} / \mathrm{v})$ lactalbumin hydrolysate and $0.002 \%(\mathrm{w} / \mathrm{v})$ phenol red, $20 \%(\mathrm{v} / \mathrm{v})$ unheated horse serum, $2.5 \%(\mathrm{v} / \mathrm{v})$ extract of baker's yeast, benzyl penicillin $(100$ i. $\mathrm{u} . / \mathrm{ml})$, and $0.05 \%(\mathrm{w} / \mathrm{v})$ thallium acetate; the $\mathrm{pH}$ was adjusted to 7.8 with $\mathrm{N}-\mathrm{NaOH}$. The agar medium was composed in the same way except that phenol red was omitted and $1 \%(\mathrm{w} / \mathrm{v})$ of Noble agar (Difco) was added.

Preparation of antisera. Rabbits were inoculated with mycoplasmas grown in liquid medium. The cultures were incubated for 3 to 4 days at $37^{\circ} \mathrm{C}$, and the organisms were harvested by centrifugation for $1 \mathrm{hr}$. at $31,000 \times \mathrm{g}$ and $4^{\circ} \mathrm{C}$. The sediment was then washed 5 times in isotonic saline. The washed sediment was resuspended in isotonic saline 1:200 of the volume of liquid medium, and the suspension was frozen and thawed from $-20^{\circ} \mathrm{C}$ to $20^{\circ} \mathrm{C} 8$ times. The suspension was then emulgated with an equal amount of Freund's complete adjuvant (Difco). Each rabbit was given $1 \mathrm{ml}$ i. $\mathrm{m}$. of the mycoplasma-adjuvant mixture and $1 \mathrm{ml}$ i.p. for 2 subsequent days and $1.5 \mathrm{ml}$ i.m. for 3 further days. After 6 weeks the rabbit was given 1.5 $\mathrm{ml}$ i.m. and serum was collected 1 week later. The sera were stored at $-20^{\circ} \mathrm{C}$. Reference antiserum to the BTS-7 strain of M. hyorhinis, produced in the donkey, was kindly supplied by J. G. Tully, National Institute of Allergy and Infectious Diseases, National Institutes of Health, Bethesda, Maryland.

Adsorption of antisera. All antisera were adsorbed by concentrated mycoplasma medium in order to remove antibodies to this medium. The mycoplasma medium was concentrated 10 times by dialysis with polyethylene-glykol in a dialysis tube. Equal amounts of concentrated mycoplasma medium and antiserum were mixed and kept at $4^{\circ} \mathrm{C}$ for $18 \mathrm{hrs}$. The sediment was removed by centrifugation for $30 \mathrm{~min}$. at $4500 \times \mathrm{g}$ and $4^{\circ} \mathrm{C}$. The clear supernatant was then concentrated to the original volume by dialysis.

Check for specificity of antisera. The adsorbed antisera were tested by gel diffusion with concentrated mycoplasma medium as antigen; the antisera did not react with this antigen. The test was carried out as previously described by Dinter et al. (1965). 
Disc growth inhibition. The technique used in this test has previously been described by Clyde (1964), but included the details described by Dinter \& Taylor-Robinson (1969).

Metabolic inhibition. This test was carried out with sterile microtitre plates as described by Taylor-Robinson et al. (1966).

Indirect haemagglutination. The technique used was that of Krogsgaard-Jensen (1971) with some modifications.. The mycoplasmas were grown in the mycoplasma medium as described above, and the antisera were the same as those used in the other tests. The supernatant of the sonicated mycoplasmas was used as antigen, and the tests were carried out with fresh, tanned sheep erythrocytes. The buffer used was phosphate-buffered saline.

Indirect epi-immunofluorescence. This test was carried out as described by Rosendal \& Black (1972) on unfixed agar colonies by indirect immunofluorescence. Eight agar blocks were incubated with antiserum in dilutions of $1: 80$ to $1: 2560$; a non-immune rabbit serum and phosphate-buffered saline were used as controls. A commercial horse anti-rabbit immunoglobulin conjugated with fluorescein isothiocyanate (Central Laboratorium van de Bloed transfusiedienst van het Nederlandsche Roode Kruis) diluted 1:10 was used. The microscope was a Leitz Ortholux with lamp housing 250 equipped with a Philips lamp type CS 15014 and a Ploem illuminator system.

\section{RESULTS}

Sensitivity of mycoplasma strains to antisera in disc growth inhibition test

All mycoplasma strains were tested against antisera to 11 strains of M. hyorhinis (BTS-7, F, FD, GDL, S, 200, K, S7, SK, $G$, and $W$ ) and the $J$ strain of $M$. suipneumoniae. In the disc growth inhibition test the inhibition pattern was very divergent (Table 1). Antiserum against the M.hyorhinis strain BTS-7 inhibited the growth of all strains except the $W$ strain. This strain was insensitive and was only inhibited by its own antiserum. Another extremely insensitive strain was the $G$ strain which was only inhibited by the BTS-7 antiserum. The most sensitive strain was the KR strain which was the only one inhibited by all 11 antisera against $M$. hyorhinis strains. Antisera to the M. hyorhinis strains S, 200, SK, and G inhibited the smallest number of strains.

Between M. suipneumoniae strain $\mathbf{J}$ and $M$.hyorhinis there were cross-reactions in as much as the $J$ strain was inhibited by antisera against the M. hyorhinis strains F, K, 200, S7, SK, and $W$. On the other hand, no M. hyorhinis strain was inhibited by antiserum to the $J$ strain. 
Table 1. Sensitivity of Mycoplasma hyorhinis and Mycoplasma suipneumoniae to inhibition by antiserum discs.

\begin{tabular}{|c|c|c|c|c|c|c|c|c|c|c|c|c|}
\hline \multirow[b]{2}{*}{ Strain } & \multirow[b]{2}{*}{ BTS-7** } & \multicolumn{11}{|c|}{ Zones of inhibition $(\mathrm{mm})^{*}$ with antisera to } \\
\hline & & $\mathbf{F}$ & FD & GDL & $\mathbf{S}$ & 200 & $\mathbf{K}$. & S7 & SK & G & $\mathbf{w}$ & $\mathbf{J}$ \\
\hline \multicolumn{13}{|l|}{ M. hyorhinis } \\
\hline BTS-7 & $2-3$ & 3 & (3) & 3 & 3 & 0 & 0 & $2-3$ & 0 & (2) & 3 & $\mathbf{0}$ \\
\hline $\mathbf{F}$ & 3.5 & 4 & 0 & 3 & 4 & $2-3$ & 3 & 3 & (4) & 0 & 4 & $\mathbf{0}$ \\
\hline FD & (2) & 0 & 3 & 0 & (3) & 0 & 3 & (3) & 0 & 0 & (4) & 0 \\
\hline GDL & 4 & 0 & 0 & 1.5 & (3) & (3) & 2 & 0 & (4) & 0 & 3 & 0 \\
\hline $\mathbf{S}$ & (2) & 2 & 3 & 3 & 3 & 0 & 0 & 3 & 0 & 0 & 4 & 0 \\
\hline 200 & 3 & 4 & $2-3$ & 2 & 0 & $2-3$ & 0 & 3 & 0 & 0 & 2 & 0 \\
\hline $\mathbf{K}$ & 2.5 & 3 & (2) & 0 & 0 & $(2.5)$ & 2.5 & 0 & 0 & 0 & $2-3$ & 0 \\
\hline S7 & 3 & (3.5) & 3 & 0 & 0 & 0 & $2-3$ & $(3.5)$ & 0 & 0 & 3 & 0 \\
\hline SK & 3 & 0 & 0 & 3 & 0 & 0 & 0 & 0 & 2 & 3 & 3 & 0 \\
\hline $\mathbf{G}$ & 1.5 & 0 & 0 & 0 & 0 & 0 & 0 & 0 & 0 & 0 & 0 & 0 \\
\hline W & 0 & 0 & 0 & 0 & 0 & 0 & 0 & 0 & 0 & 0 & (2) & 0 \\
\hline KR & 3 & 4 & $4-5$ & 3 & 6 & 7 & 3 & $5-6$ & 4 & 2 & $3-4$ & 0 \\
\hline 872 & 3 & 4 & 4 & 4 & 0 & 2 & $2-3$ & 5 & $3-4$ & 3 & 6 & 0 \\
\hline 378 & (2) & (2) & 2 & $(2-3)$ & 0 & 0 & 2 & $2-3$ & $2-3$ & 2 & 2 & 0 \\
\hline 859 & 2 & 3 & $1-2$ & 0 & 2 & 3 & $2-3$ & 2 & 2 & 0 & 0 & 0 \\
\hline 959 & (2) & $1-2$ & 0 & 2 & (2) & 0 & $2-3$ & $1-2$ & 0 & 3 & $2-3$ & $\mathbf{0}$ \\
\hline 201 & $3-4$ & 3 & 2 & $(2)$ & 0 & 0 & $2-3$ & 0 & 0 & 0 & 3 & 0 \\
\hline 203 & $2-3$ & 0 & 2 & 0 & 0 & 0 & 0 & 0 & (2) & 0 & 0 & 0 \\
\hline M. sui- & & & & & & & & & & & & \\
\hline pneumoniae $\mathrm{J}$ & $\begin{array}{ll}\mathbf{J} & 0\end{array}$ & 2 & 0 & 0 & 0 & 2 & 3 & $1-2$ & 3 & 0 & (2) & 2 \\
\hline
\end{tabular}

* Zones less than $1 \mathrm{~mm}$ in diameter are indicated with 0 .

* Reference serum.

Figures in brackets indicate solitary colonies of mycoplasmas in the zone of inhibition.

Metabolic inhibition test (MIT)

All strains were tested against antisera to M. hyorhinis strains GDL, S, 200, K, G, and W, and M. suipneumoniae strain J (Table 2). In disc growth inhibition test, antisera GDL, $K$, and $W$ inhibited a large number of strains whereas antisera $S, 200$, and $G$ inhibited a small number of strains. When the antisera were tested in MIT, 5 strains (SK, 872, 378, 859, and 203) were not inhibited by antiserum 200, whereas 1 strain (959) was inhibited, but not by all antisera and even then only at a low serum dilution. There was a difference between titres obtained with the "old" strains and the "fresh" isolates (Table 2). The "old" strains reacted with nearly all the antisera at high serum dilutions whereas the "fresh" isolates reacted mainly at low serum 
Table 2. Sensitivity of Mycoplasma hyorhinis and Mycoplasma suipneumoniae to rabbit antisera in the metabolic inhibition test.

\begin{tabular}{|c|c|c|c|c|c|c|c|}
\hline \multirow[b]{2}{*}{ Strain* } & \multicolumn{7}{|c|}{ Reciprocal titres of rabbit antisera to } \\
\hline & GDL & $\mathbf{S}$ & 200 & $\mathbf{K}$ & G & $\mathbf{w}$ & $\mathbf{J}$ \\
\hline \multicolumn{8}{|l|}{ M. hyorhinis } \\
\hline F & 1280 & 1280 & 2560 & 2560 & 640 & 1280 & $<10$ \\
\hline FD & 640 & 1280 & 160 & 1280 & 320 & 2560 & $<10$ \\
\hline GDL & 1280 & 1280 & 320 & 640 & 640 & 2560 & $<10$ \\
\hline $\mathbf{S}$ & 1280 & 1280 & 1280 & 1280 & 320 & 2560 & $<10$ \\
\hline 200 & 20 & 2560 & 320 & 2560 & 320 & 2560 & $<10$ \\
\hline $\mathbf{K}$ & 640 & 2560 & 1280 & 1280 & 160 & 2560 & $<10$ \\
\hline S7 & 1280 & 2560 & 160 & 2560 & 640 & 1280 & $<10$ \\
\hline SK & 160 & 1280 & $<10$ & 640 & 2560 & 320 & $<10$ \\
\hline $\mathbf{G}$ & 640 & 1280 & 320 & 640 & 640 & 2560 & $<10$ \\
\hline W & 2560 & 2560 & 1280 & 2560 & 1280 & 2560 & $<10$ \\
\hline $\mathrm{KR}$ & 20 & 320 & 80 & 160 & 20 & 640 & $<10$ \\
\hline 872 & 80 & 1280 & $<10$ & 1280 & 40 & 80 & $<10$ \\
\hline 378 & 640 & 40 & $<10$ & 10 & 320 & 160 & $<10$ \\
\hline 859 & 20 & 640 & $<10$ & 640 & 20 & 80 & $<10$ \\
\hline 959 & 20 & $<10$ & 10 & 80 & 10 & 80 & $<10$ \\
\hline 201 & 2560 & 80 & 40 & 640 & 80 & 1280 & $<10$ \\
\hline 203 & 40 & 1280 & $<10$ & 640 & 20 & 320 & $<10$ \\
\hline $\begin{array}{l}\text { M. sui- } \\
\text { pneumoniae J }\end{array}$ & $<10$ & $<10$ & $<10$ & $<10$ & $<10$ & $<10$ & 20 \\
\hline
\end{tabular}

" M.. hyorhinis strains F through W are all "old" strains, whereas all the other strains are "fresh" isolates (see Materials and Methods).

dilutions. M. suipneumoniae was not inhibited by any of the M. hyorhinis antisera, and the M. suipneumoniae antiserum did not inhibit any of the tested M. hyorhinis strains.

\section{Indirect haemagglutination (IHA)}

Seventeen strains of M. hyorhinis and a strain of M. suipneumoniae were tested in IHA to $10 \mathrm{M}$. hyorhinis antisera and $1 \mathrm{M}$. suipneumoniae antiserum (Table 3). The antiserum to M.hyorhinis strain GDL did not give positive reactions with 4 other strains of the same species (F, FD, G, and W), nor the antiserum to the S7 strain with the FD and $W$ strains. The haemagglutination by the $\mathrm{K}$ and 378 strains was observed only at low serum dilutions throughout. M. suipneumoniae gave either no haemagglutination or only reactions with M. hyorhinis antisera at very low dilutions. 
T a b l e 3. Cross-reactions in indirect haemagglutination between Mycoplasma hyorhinis strains and Mycoplasma suipneumoniae.

\begin{tabular}{|c|c|c|c|c|c|c|c|c|c|c|c|}
\hline \multirow[b]{2}{*}{ Strain } & \multicolumn{11}{|c|}{ Reciprocal titres of rabbit antisera to } \\
\hline & $\mathbf{F}$ & FD & GDL & $\mathbf{S}$ & 200 & $\mathbf{K}$ & S7 & SK & G & $\mathbf{w}$ & $\mathbf{J}$ \\
\hline \multicolumn{12}{|l|}{ M. hyorhinis } \\
\hline F & 2048 & 1024 & $<2$ & 32 & 16 & 32 & 64 & 128 & 64 & 128 & $<2$ \\
\hline FD & 2048 & 2048 & $<2$ & 16 & 32 & 32 & $<2$ & 256 & 64 & 128 & $<2$ \\
\hline GDL & 512 & 256 & 64 & 256 & 64 & 512 & 128 & 128 & 128 & 256 & $<2$ \\
\hline $\mathbf{S}$ & 512 & 128 & 128 & 256 & 128 & 512 & 256 & 64 & 128 & 512 & 2 \\
\hline 200 & 512 & 1024 & 64 & 512 & 512 & 512 & 512 & 256 & 512 & 512 & $<2$ \\
\hline $\mathbf{K}$ & 128 & 64 & 64 & 64 & 64 & 64 & 64 & 64 & 32 & 128 & $<2$ \\
\hline S7 & 512 & 256 & 32 & 256 & 16 & 128 & 128 & 32 & 128 & 1024 & $<2$ \\
\hline SK & 2048 & 1024 & 32 & 128 & 256 & 32 & 64 & 512 & 128 & 512 & $<2$ \\
\hline $\mathbf{G}$ & 512 & 256 & $<2$ & 32 & 32 & 128 & 32 & 32 & 32 & 64 & $<2$ \\
\hline W & 4096 & 32 & $<2$ & 32 & 16 & 64 & $<2$ & 64 & 64 & 128 & $<2$ \\
\hline KR & 256 & 1024 & 128 & 256 & 1024 & 512 & 256 & 512 & 256 & 1024 & 8 \\
\hline 872 & 256 & 256 & 128 & 512 & 256 & 512 & 512 & 256 & 256 & 2048 & 4 \\
\hline 378 & 128 & 256 & 64 & 64 & 64 & 128 & 128 & 64 & 64 & 128 & 8 \\
\hline 859 & 256 & 512 & 512 & 512 & 256 & 512 & 512 & 1024 & 256 & 1024 & 4 \\
\hline 959 & 512 & 1024 & 128 & 512 & 1.024 & 512 & 512 & 256 & 256 & 1024 & 4 \\
\hline 201 & 256 & 512 & 128 & 256 & 512 & 512 & 256 & 256 & 256 & 1024 & $<2$ \\
\hline 203 & 128 & 256 & 256 & 256 & 256 & 128 & 128 & 128 & 128 & 512 & 2 \\
\hline \multicolumn{12}{|l|}{ M. sui- } \\
\hline pneumoniae $\mathbf{J}$ & $<2$ & $<2$ & $<2$ & $<2$ & $<2$ & 4 & $<2$ & 2 & $<2$ & $<2$ & 64 \\
\hline
\end{tabular}

Indirect epi-immunofluorescence (IF)

Eighteen strains of $M$. hyorhinis and a strain of M. suipneumoniae were tested in IF with $10 \mathrm{M}$. hyorhinis antisera and the M. suipneumoniae antiserum (Table 4). All the M.hyorhinis strains gave strong immunofluorescence to a homologous antiserum in a high dilution, but did not react in any case with the M. suipneumoniae antiserum. M. suipneumoniae gave only IF with antiserum to the homologous strain. The IF was the test that gave the most homogenous results. The difference in titres obtained is partly due to dissimilarities in size and structure of the mycoplasma colonies.

\section{DISCUSSION}

In this study serological differentiation between $M$. hyorhinis and M. suipneumoniae was carried out by disc growth inhibition, metabolic inhibition, indirect haemagglutination and in- 
Table 4. Antigenic relationship of Mycoplasma hyorhinis and Mycoplasma suipneumoniae in the indirect epi-immunofluorescence technique.

\begin{tabular}{|c|c|c|c|c|c|c|c|c|c|c|c|}
\hline \multirow[b]{2}{*}{ Strain } & \multicolumn{11}{|c|}{ Reciprocal titres of rabbit antisera to } \\
\hline & $\mathbf{F}$ & FD & GDL & $\mathbf{S}$ & 200 & $\mathbf{K}$ & S7 & SK & G & $\mathbf{w}$ & J \\
\hline \multicolumn{12}{|l|}{ M. hyorhinis } \\
\hline BTS-7 & 1280 & 2560 & 1280 & 1280 & 1280 & 640 & 2560 & 1280 & 640 & 1280 & $<80$ \\
\hline F & 1280 & 640 & 160 & 320 & 320 & 1280 & 2560 & 320 & 640 & 1280 & $<80$ \\
\hline FD & 640 & 640 & 160 & 640 & 640 & 640 & 640 & 640 & 32.0 & 640 & $<80$ \\
\hline GDL & 1280 & 1280 & 320 & 320 & 320 & 1280 & 320 & 640 & 640 & 640 & $<80$ \\
\hline $\mathbf{S}$ & 1280 & 1280 & 320 & 1280 & $32: 0$ & 1280 & 2560 & 320 & 1280 & 1280 & $<80$ \\
\hline 200 & 2560 & 2560 & 1280 & 1280 & 1280 & 1280 & 1280 & 128.0 & 1280 & 1280 & $<80$ \\
\hline K & 320 & 320 & 320 & 160 & 160 & 640 & 2560 & 320 & 320 & 640 & $<80$ \\
\hline S7 & 320 & 640 & 640 & 640 & 1280 & 1280 & 1280 & 320 & 640 & 640 & $<80$ \\
\hline SK & 1280 & 1280 & 1280 & 2560 & 1280 & 1280 & 1280 & 2560 & 1280 & 640 & $<80$ \\
\hline G & 1280 & 640 & 320 & 640 & 320 & 1280 & 640 & 1280 & 640 & 1280 & $<80$ \\
\hline W & 1280 & 2560 & 1280 & 2560 & 1280 & 1280 & 1280 & 1280 & 1280 & 1280 & $<80$ \\
\hline KR & 64.0 & 1280 & 1280 & 1280 & 1280 & 6410 & 1280 & 1280 & 160 & 1280 & $<80$ \\
\hline 872 & 1280 & 1280 & 1280 & 1280 & 128.0 & 1280 & 640 & 640 & 1280 & 1280 & $<80_{>}$ \\
\hline 378 & 1280 & 1280 & 1280 & 1280 & 640 & 1280 & 2560 & 640 & 1280 & 2560 & $<80\rangle$ \\
\hline 859 & 1280 & 1280 & 1280 & 1280 & 1280 & 1280 & 1280 & 1280 & 640 & 1280 & $\langle 80\rangle$ \\
\hline 959 & 640 & 640 & 320 & 640 & 1280 & 1280 & 1280 & 640 & 640 & 1280 & $<80\rangle$ \\
\hline 201 & 640 & 1280 & 1280 & 1280 & 640 & 1280 & 1280 & 640 & 640 & 1280 & $<80\rangle$ \\
\hline 203 & 1280 & 1280 & 320 & 1280 & 640 & 320 & 640 & 320 & 320 & 640 & $<80\rangle$ \\
\hline $\begin{array}{l}\text { M. sui- } \\
\text { pneumoniae J }\end{array}$ & $<80$ & $<80$ & $<80$ & $<80$ & $<80$ & $<80$ & $<80$ & $<80$ & $<80$ & $<80$ & 320 \\
\hline
\end{tabular}

direct epi-immunofluorescence. Only in disc growth inhibition was the growth of $M$. suipneumoniae inhibited by some M. hyorhinis antisera (Table 1). However, it is impossible to say why such cross-reactions occurred.

Disc growth inhibition showed the most irregular pattern of all the tests. Dinter \& Taylor-Robinson (1969) were able to divide 9 strains of $M$. hyorhinis into sensitive and non-sensitive groups based on the inhibition of growth by antisera. However, it became obvious that such a grouping is not consistently reproducible, i. e., when the very same strains of $M$. hyorhinis were tested on different occasions (cf. the present results with those of Dinter et al. 1965, and Dinter \& Taylor-Robinson). The inhibition of growth results from the combination of antibody with specific reactive sites of the cell surface. Consequently, the inconsistent results of disc growth inhibition might be an expression of fluctuating changes in the composition of the myco- 
plasmal membrane, changes probably induced by different growing conditions on non-synthetic media. It must be admitted, however, that also in our tests 2 strains, $G$ and $W$, were remarkably resistant to inhibition while 2 other strains, $K R$ and 872 , were remarkably sensitive (Table 1 ).

In a study by Roberts \& Pijoan (1971), it was found that disc growth inhibition could be used for testing both laboratory strains and recent isolates of $M$. hyorhinis by using fresh horse serum instead of commercial horse serum. By using their method no changes in the irregular pattern of growth inhibition could be obtained. It is therefore concluded that disc growth inhibition is an unsuitable test for typing M. hyorhinis and for differentiating that species from M. suipneumoniae.

Both in the metabolic inhibition test (MIT) and the indirect haemagglutination test, some strains of $M$.hyorhinis failed to react with antisera to some other strains. This problem might be solved by using an antiserum which has given strong reactions with all the tested strains, but there is no evidence that such an antiserum would react with all $M$. hyorhinis strains on every occasion. It is worth mentioning that in MIT the "old" strains were much more sensitive to antisera than the "fresh" isolates, but the nature of this discrepancy cannot be explained. In short, the MIT and the indirect haemagglutination test are not suitable for the differentiation of M. hyorhinis from M. suipneumoniae.

The indirect epi-immunofluorescence would appear to be the most suitable method for typing porcine mycoplasmas, because it gives distinct titres and no reactions which are difficult to interpret.

\section{ACKNOWLEDGEMENTS}

Thanks are due to Drs. H. Ernф, N. F. Friis, A. Meyling, and S. Rosendal for valuable discussions concerning the growing and typing of mycoplasmas, and to Miss Margareta Horn af Rantzien for her excellent technical assistance.

\section{REFERENCES}

Clyde, Jr., W. A.: Mycoplasma species identification based upon growth inhibition by specific antisera. J. Immunol. 1964, 92, 958-965.

Dinter, Z., D. Danielsson \& K. Bakos: Differentiation of porcine mycoplasma strains. J. gen. Microbiol. 1965, 41, 77-84. 
Dinter, Z. \& D. Taylor-Robinson: Susceptibility and resistance of various strains of Mycoplasma hyorhinis to antisera, polymyxins and low $\mathrm{pH}$ values. J. gen. Microbiol. 1969, 57, 263-272.

Krogsgaard-Jensen, A.: Indirect hemagglutination with mycoplasma antigens: effect of $\mathrm{pH}$ on antigen sensitization of tanned fresh and formalinized sheep erythrocytes. Appl. Microbiol. 1971, 22, $756-759$.

Roberts, D. H. \& C. Pijoan: Identification of Mycoplasma hyorhinis. Brit. vet. J. $1971,127,582-586$.

Rosendal, $S$. \& F. T. Black: Direct and indirect immunofluorescence of unfixed and fixed mycoplasma colonies. Acta path. microbiol. scand. Section B 1972, 80, 615-622.

Taylor-Robinson, D., R. H. Purcell, D. C. Wong \& R. M. Chanock: A colour test for the measurement of antibody to certain mycoplasma species based upon the inhibition of acid production. J. Hyg. (Lond.) 1966, 64, 91-105.

\section{SAMMANFATTNING}

Utvärdering av några serologiska metoder för identifiering av Mycoplasma hyorhinis och Mycoplasma suipneumoniae.

Aderton stammar av Mycoplasma hyorhinis och en stam av Mycoplasma suipneumoniae har testats med följande serologiska metoder: växtinhibition på fast substrat, växtinhibition i flytande substrat, indirekt hemagglutination och indirekt epi-immunofluorescens. Endast med epi-immunofluorescens var det möjligt att klassificera alla undersökta stammar med avseende på art. De övriga metoderna visade sig vara mindre pålitliga eftersom reaktionen mellan testsera och vissa stammar var svag eller negativ. Dessutom förekom korsreaktioner mellan M. suipneumoniae och vissa stammar av M. hyorhinis.

(Received December 22, 1973).

Reprints may be requested from: Rolf Jansson, National Veterinary Institute, S-104 05 Stockholm 50, Sweden. 